\title{
Ge-Al Multilayer Thin Film as an Anode for Li-ion Batteries
}

\author{
Jae-Young Lee ${ }^{a}$, Duc Tung Ngo ${ }^{\text {a }}$, and Chan-Jin Park ${ }^{\dagger}$ \\ Department of Materials Science and Engineering, Chonnam National University, Gwangju 61186, Korea
}

(Received April 12, 2017; Accepted May 17, 2017)

\begin{abstract}
We design $\mathrm{Ge}-\mathrm{Al}$ multilayer assemblies as anode materials for Li-ion batteries, in which $\mathrm{Ge}$ and $\mathrm{Al}$ thin films are alternately deposited by a radio sputtering method. By sandwiching Ge layers between Al layer, the cyclability, rate capability, and capacity of Ge are improved significantly. The success of the $\mathrm{Ge}-\mathrm{Al}$ multilayer is attributed to the Al films. To maintain the integrity of electrical contact, $\mathrm{Al}$ acts as an elastic layer, which can expand or shrink with the Ge film upon lithiation or delithiation. In addition, the presence of the $\mathrm{Al}$ film on the surface can prevent direct contact of Ge and electrolyte, thereby reducing the growth of a SEI layer. Importantly, with high electrical and ionic conductivities, the Al film provides efficient electrical and ionic routes for electrons and Li-ions to access the Ge film, promoting a high specific capacity and high rate capability for Ge.
\end{abstract}

Key words : Batteries, Thin films, Germanium, Aluminum, Cyclability

\section{Introduction}

$\mathrm{H}^{\mathrm{i}}$ gh-energy-density Li-ion batteries (LIBs) are necessary for portable electronics, electric vehicles, and energy storage systems. ${ }^{1)}$ In addition, the rising demand for wearable electronic devices has led to the development of thin and small batteries. ${ }^{2,3)}$ LIBs have been widely used for many applications because they offer high energy density, negligible self-discharge, and long cycle life. Thus far, graphite has been widely used as an anode material for commercial LIBs because of its unique properties such as low cost and high stability.,5) Unfortunately, its theoretical capacity is only $372 \mathrm{mAh} \mathrm{g}^{-1}$, which is unsuitable for high-power and highenergy-density LIBs. In order to increase the capacity of LIBs, the replacement of graphite with a new advanced material having a higher specific capacity is necessary.

In this context, Li alloy-based anode materials such as $\mathrm{Si}$, Ge, and Sn have received much attention because they have extremely high capacities and low potential for alloying/ dealloying. ${ }^{6-9)}$ Among these materials, Ge exhibits superior electrochemical performance over Si and Sn. Si has a higher specific capacity compared to that of Ge, but Ge remains more attractive than $\mathrm{Si}$ because the electrical conductivity of $\mathrm{Ge}$ is $10^{4}$ times higher than that of Si. In addition, at room temperature, the Li-ion diffusivity of bulk Ge is 400 times higher than that of $\mathrm{Si}^{10}{ }^{10}$ In comparison with $\mathrm{Sn}, \mathrm{Ge}$ dominates over $\mathrm{Sn}$ in all aspects, including specific capacity, stability, and rate capability. ${ }^{11)}$ Although Sn is metallic with a very high electrical conductivity, the very low Li-ion diffu-

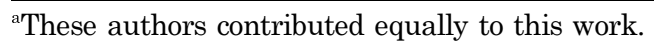

Corresponding author: Chan-Jin Park

E-mail : parkcj@jnu.ac.kr

Tel : +82-62-530-1704 Fax : +82-62-530-1699
}

sivity in bulk $\mathrm{Sn}$ impedes its performance. ${ }^{12)}$ Despite its merits, Ge undergoes considerable volume changes, which can cause fast cracking and pulverization of the electrode, leading to mechanical stress, structural damage, and resulting capacity loss after a few cycles. To solve this problem, most previous studies have focused on using buffer layers to mitigate internal stresses, using amorphous Ge to prevent anisotropic expansion, and reducing the particle size in order to minimize the volume expansion. ${ }^{13,14)}$ For thin-film batteries, to minimize the stress caused by significant volume expansion, multilayer electrodes have been widely used, in which the active layer and buffer layer are alternated together. ${ }^{15}$

$\mathrm{Al}$ is a very attractive buffer material because of its superior electrical conductivity, light weight, abundance, environmental friendliness, and recyclability. Therefore, it is expected that the addition of $\mathrm{Al}$ to Ge thin films can improve the electrical performance of the Ge films. This is attributed to the high electrical conductivity of $\mathrm{Al}$, which may improve the electrical conductivity of the Ge film and thereby lower the electrode polarization. In addition, because $\mathrm{Al}$ has high $\mathrm{Li}$ ionic conductivity, the $\mathrm{Al}$ film may promote Li-ion diffusion, consequently facilitating the ionic conductivity of Ge film. Most importantly, the presence of the $\mathrm{Al}$ layer can act as an elastic layer to preserve the Ge film during alloying and maintain the integrity of the electrical contact between Ge and the current collector.

In this work, we propose a new anode concept in which the Ge film is sandwiched between $\mathrm{Al}$ layers by radio-frequency (RF) sputtering. The multilayer $\mathrm{Ge}-\mathrm{Al}$ outperforms pure Ge in terms of cyclability and rate capability. 


\section{Experimental Procedure}

\subsection{Material preparation}

The Ge-Al multilayer electrodes were deposited directly on a $\mathrm{Cu}$ foil (Iljin Copper Corporation) as a current collector by an RF sputtering method from Ge (Toshima, Japan, 99.99\%) and $\mathrm{Al}$ (Materion, USA, 99.99\%) targets. Briefly, the $\mathrm{Ge}-\mathrm{Al}$ multilayer was deposited sequentially. Firstly, the chamber was evacuated to $3.75 \times 10^{-6}$ Torr as a base pressure. High-purity Ar (99.999\%) was introduced to the chamber before sputtering and the working pressure of $3 \times$ $10^{-3}$ Torr was maintained by controlling the Ar flow rate to $20.0 \mathrm{sccm}$. The sputtering power was maintained at $\sim 80 \mathrm{~W}$ for both the Ge and $\mathrm{Al}$ targets, and the distance between the $\mathrm{Cu}$ substrate and the sputtering target was $10 \mathrm{~cm}$. By controlling the sputtering time, we easily controlled the thicknesses of the $\mathrm{Ge}$ and $\mathrm{Al}$ films. In this work, the total thickness of the $\mathrm{Ge}-\mathrm{Al}$ multilayer was fixed to $\sim 300 \mathrm{~nm}$, while the thickness of either Ge or Al was changed. Notably, the thickness ratio of Ge:Al was held constant at $~ 5: 1$ for all experiments. Details on the thickness and number of layers of $\mathrm{Ge}$ and $\mathrm{Al}$ are given in Table 1.

\subsection{Physical characterization}

The morphologies of the samples were investigated using a field-emission scanning electron microscope (FE-SEM, S4700/EX-200, Hitachi, Japan). Cross-sectional images of the $\mathrm{Ge}-\mathrm{Al}$ multilayers were observed with transmission electron microscopy (TEM, TECNAL-G ${ }^{2}$ ) using focused ion beam (FIB) milled samples. Energy-dispersive X-ray spectroscopy (EDS) was performed to analyze the composition of the Ge-Al electrodes. The Ge films and Ge-Al multilayers were structurally characterized with a multipurpose X-ray diffractometer (XRD, X'Pert PRO) using $\mathrm{Cu}$ Ka radiation.

\subsection{Electrochemical characterization}

To investigate the electrochemical properties of the $\mathrm{Ge}-\mathrm{Al}$ and pure Ge electrodes, the samples were punched into 14mm-diameter disks. Using the punched disk electrodes, 2032-type coin cells were assembled in an Ar-filled glove box, with $\mathrm{Li}$ metal as a counter electrode and a glass fiber soaked with $1 \mathrm{M} \mathrm{LiPF}_{6}$ in ethylene carbonate (EC) and dimethyl carbonate (DMC) (1:1 by volume) as the separator. The electrochemical properties of the $\mathrm{Ge}-\mathrm{Al}$ and Ge electrodes were investigated using cyclic voltammetry (CV) in the potential range of 0.0 to $2 \mathrm{~V}$ vs. $\mathrm{Li} / \mathrm{Li}^{+}$at a scan rate of $0.2 \mathrm{mV} \mathrm{s}^{-1}$ with a potentiostat (Gamry-PC750). Further, the electrodes were galvanostatically charged and discharged between 0.01 and $1.5 \mathrm{~V}$ vs. $\mathrm{Li} / \mathrm{Li}^{+}$using an automatic battery cycle (WonATech-WBCS3000). All the experiments were conducted at $25^{\circ} \mathrm{C}$. Electrochemical impedance spectroscopy (EIS) measurements were conducted in the frequency range from $10 \mathrm{mHz}$ to $100 \mathrm{KHz}$.

\section{Results and Discussion}

Figure 1 presents the XRD patterns of the Ge and $\mathrm{Ge}-\mathrm{Al}$ samples. To eliminate the effect of the $\mathrm{Cu}$ substrate, XRD

Table 1. Thickness and Number of Layers in Each Ge-Al Multilayer

\begin{tabular}{|c|c|c|c|c|c|}
\hline \multirow{2}{*}{$\begin{array}{c}\text { Total number } \\
\text { of layers }\end{array}$} & \multicolumn{2}{|c|}{$\mathrm{Ge}$} & \multicolumn{2}{|c|}{$\mathrm{Al}$} & \multirow{2}{*}{$\begin{array}{l}\text { Total } \\
\text { thickness } \\
\quad(\mathrm{nm})\end{array}$} \\
\hline & $\begin{array}{l}\text { Thickness } \\
\text { (nm) }\end{array}$ & $\begin{array}{l}\text { Number of } \\
\text { layers }\end{array}$ & $\begin{array}{c}\text { Thickness } \\
\text { (nm) }\end{array}$ & $\begin{array}{c}\text { Number of } \\
\text { layers }\end{array}$ & \\
\hline $4(\mathrm{Ge}-\mathrm{Al}-4)$ & 125 & 2 & 25 & 2 & \multirow{4}{*}{300} \\
\hline $10(\mathrm{Ge}-\mathrm{Al}-10)$ & 50 & 5 & 10 & 5 & \\
\hline $20(\mathrm{Ge}-\mathrm{Al}-20)$ & 25 & 10 & 5 & 10 & \\
\hline $30(\mathrm{Ge}-\mathrm{Al}-30)$ & 16.67 & 15 & 3.33 & 15 & \\
\hline
\end{tabular}
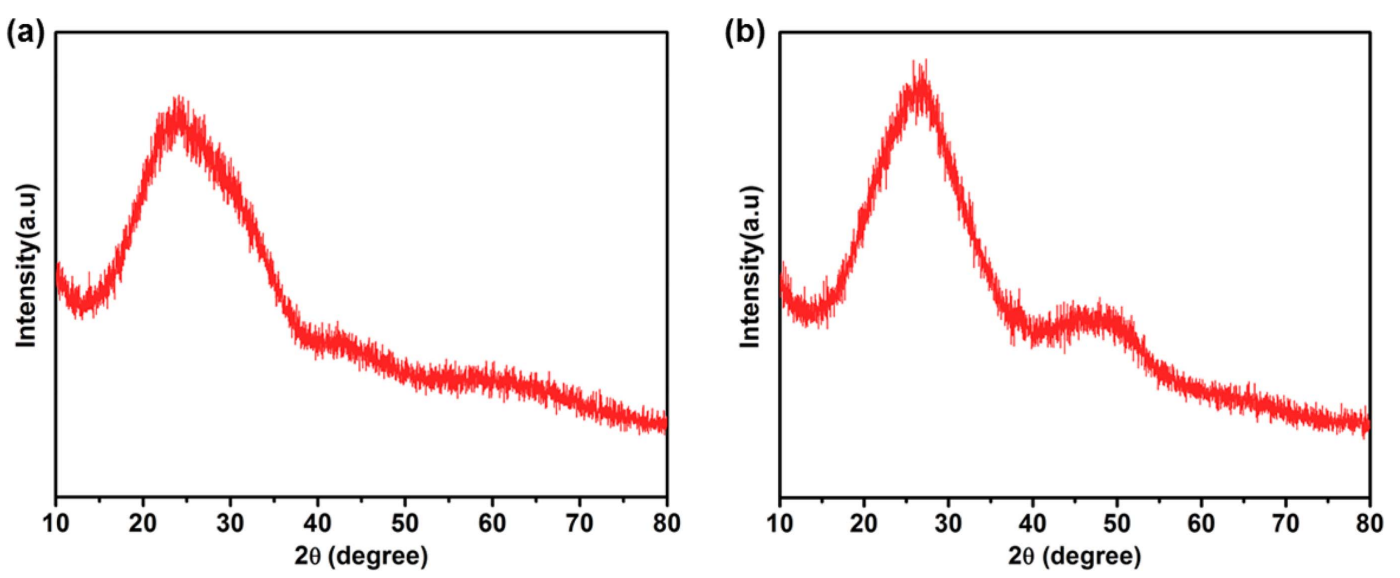

Fig. 1. X-ray diffraction (XRD) patterns of (a) pure Ge and (b) Ge-Al-20 deposited on glass. 

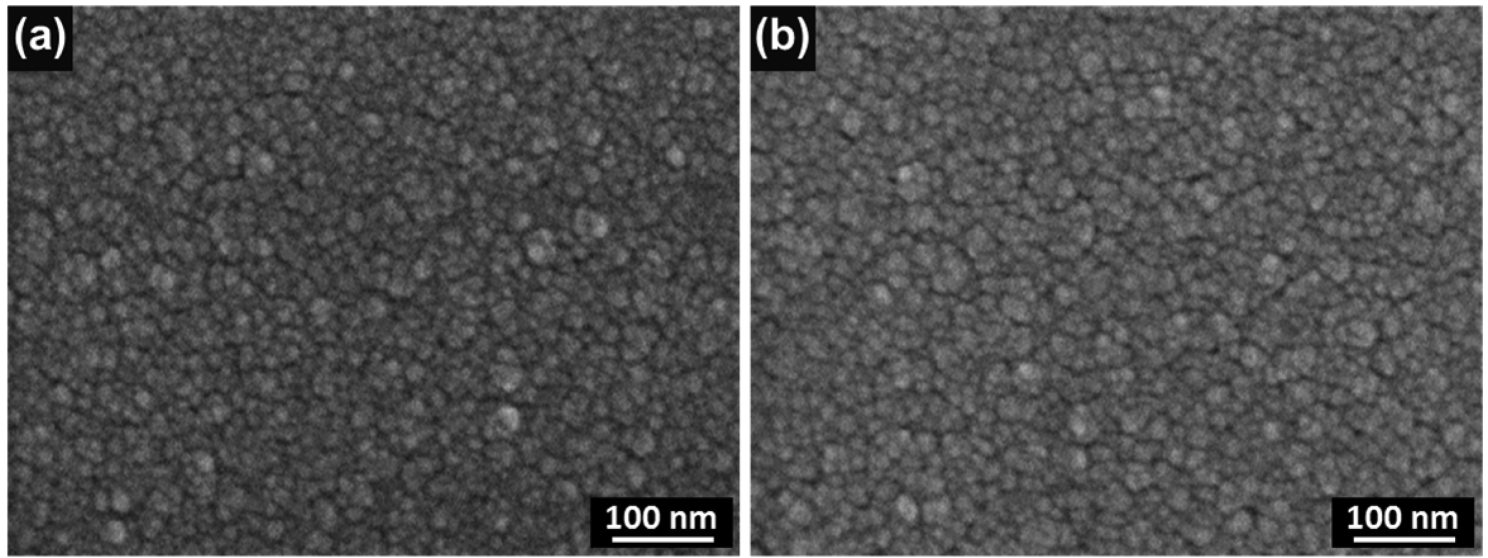

Fig. 2. Surface morphologies of (a) pure Ge and (b) Ge-Al-20.

was performed using $\mathrm{Ge}$ and $\mathrm{Ge}-\mathrm{Al}$ samples deposited on glass substrates. The obtained Ge film exhibits a very broad diffraction peak with no detected peaks of Ge, indicating that the Ge film obtained by sputtering has an amorphous structure. Similarly, Ge-Al shows the similar pattern as that of the pure Ge film. The amorphous structure is preferable for anode materials for LIBs because it can avoid anisotropic volume expansion. Amorphous structures are usually more open than well-defined crystalline structures, which can assist fast lithiation/delithiation.

The morphologies of all the samples were investigated by SEM, with results shown in Fig. 2. The pure Ge film is deposited uniformly and covers the overall surface of the $\mathrm{Cu}$ substrate, so it can maintain intimate contact with the current collector during cycling and thereby facilitate the electrochemical performance of the battery. The SEM image of pure Ge reveals small coalesced Ge grains and a densely packed morphology. Similarly, the thin film of Ge-Al-20 shows excellent coverage on the $\mathrm{Cu}$ substrate with neither grain boundaries nor defects. The Ge-Al-20 multilayer thin film shows a rough surface, because the surface profile of the $\mathrm{Cu}$ foil is reflected in the morphology of the $\mathrm{Ge}-\mathrm{Al}$ thin film.

To illustrate further the morphology of the Ge-Al multilayer, a selected $\mathrm{Ge}-\mathrm{Al}-20$ sample was further investigated by FIB milling and TEM. Fig. 3 shows the TEM image of
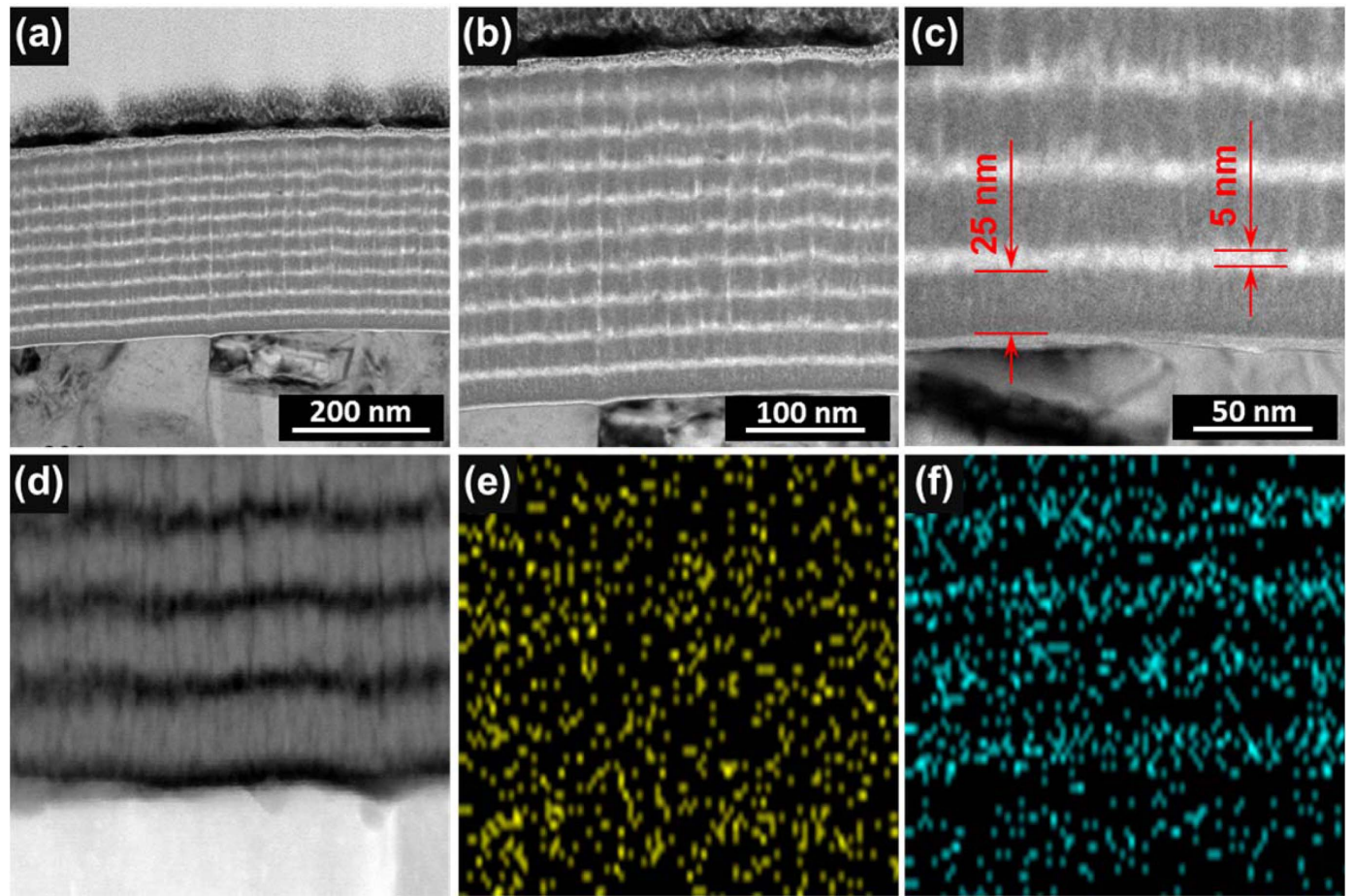

Fig. 3. (a-c) TEM images of Ge-Al-20 with various magnifications; (d) scanning transmission electron microscopy (STEM) image of Ge-Al-20, corresponding to Fig. 3(c); TEM elemental mapping of (e) $\mathrm{Al}$ and (f) Ge in Ge-Al-20. 
Ge-Al-20. The uniform Ge layer, characterized as a dark gray layer, is sandwiched between very thin Al layers, which are characterized by brittle layers. As observed in Fig. 3(a) - (c), both Ge and Al layers show uniformity over the entire sample. This result suggests the successful deposition of the Ge-Al multilayer by RF sputtering. The thickness of each $\mathrm{Ge}$ and $\mathrm{Al}$ layer was measured, as indicated in Fig. 3(c). Accordingly, the thicknesses of the Ge and Al layers are $\sim 25$ and $\sim 5 \mathrm{~nm}$, respectively, and the total thickness of Ge-Al-20 is estimated as $\sim 300 \mathrm{~nm}$. TEM elemental mapping was further performed to confirm the distribution of $\mathrm{Ge}$ and $\mathrm{Al}$ in $\mathrm{Ge}-\mathrm{Al}-20$. Because of the thinness of the $\mathrm{Al}$ layer, the distribution of $\mathrm{Al}$ does not show a perfect line, as in the TEM image. By contrast, the elemental mapping of Ge shows a clearly distinguished layer, indicated in Fig. 3(f).

To investigate the lithiation and delithiation mechanisms in the $\mathrm{Ge}$ and $\mathrm{Ge}-\mathrm{Al}$ electrodes, CVs for the pure $\mathrm{Ge}$ and $\mathrm{Ge}-\mathrm{Al}$ electrodes were measured at the potential range of $\sim 0-1.5 \mathrm{~V}$ vs. $\mathrm{Li} / \mathrm{Li}^{+}$starting from the open circuit potential (OCP) at the scan rate of $0.02 \mathrm{mV} \mathrm{s}^{-1}$. The results are shown in Fig. 4. For the Ge electrode, from OCP to $1.2 \mathrm{~V} \mathrm{vs.} \mathrm{Li} / \mathrm{Li}^{+}$, the current density is very small, suggesting that no electrochemical reaction occurs in this potential range. A minor peak appears at $1.1 \mathrm{~V} \mathrm{vs.} \mathrm{Li} / \mathrm{Li}^{+}$; the presence of this peak can be attributed to the reaction of the electrolyte with Ge. ${ }^{16)}$ Notably, the intensity of this peak is very low, indicating that the $\mathrm{Li}$ consumption for this reaction was small; this could induce good coulombic efficiency in the first cycle. Then, the current begins to increase at $\sim 0.8 \mathrm{~V} \mathrm{vs.} \mathrm{Li} / \mathrm{Li}^{+}$, and a first sharp cathodic current peak is observed at $0.59 \mathrm{~V}$ vs. $\mathrm{Li} / \mathrm{Li}^{+}$(Fig. 4(a)). The presence of this cathodic current peak is associated with the decomposition of $\mathrm{GeO}_{2}$ on the surface of the electrode and the formation of a solid electrolyte interphase (SEI) layer. However, from the second cycle onward, this peak disappears. This is because of the formation of a stable SEI layer and the $\mathrm{GeO}_{2}$ layer mostly decomposes in the first cycle. Moreover, three additional cathodic current peaks corresponding to lithiation are observed at
$0.46,0.37$, and $0.12 \mathrm{~V}$ vs. $\mathrm{Li} / \mathrm{Li}^{+}{ }^{+17)}$ respectively, in the first cathodic curve for the Ge electrode. Subsequently, in the reverse scan corresponding to the delithiation process, three anodic current peaks appear during the first anodic scan at $0.38,0.49$, and $0.70 \mathrm{~V}$ vs. $\mathrm{Li} / \mathrm{Li}^{+}$. These peaks are assigned to the delithiation of $\mathrm{Li}$ from the $\mathrm{Li}_{\mathrm{x}} \mathrm{Ge}$ alloy. It can be seen that all the anodic and cathodic curves are perfectly superimposed for all cycles, suggesting the good reversibility of the pure Ge film. Fig. 4(b) shows that the CV of the Ge-Al20 electrode exhibits similar electrochemical behaviors to that for the pure Ge film electrode. However, the minor peak at $1.1 \mathrm{~V}$ vs. $\mathrm{Li} / \mathrm{Li}^{+}$, related to the reaction between $\mathrm{Ge}$ and electrolyte in the first cycle, is absent. Notably, for the Ge-Al-20 sample, the top layer is $\mathrm{Al}$. The absence of this peak can be attributed to the low reactivity of $\mathrm{Al}$ with the electrolyte at this potential. This implies that $\mathrm{Al}$ is less active than Ge.

To examine the cyclability of pure Ge and the $\mathrm{Ge}-\mathrm{Al}$ multilayer, working electrodes of $\mathrm{Ge}$ and $\mathrm{Ge}-\mathrm{Al}$ were assembled into coin-type half-cells using $\mathrm{Li}$ metal as a counter/reference electrode. The cells were charged and discharged at the rate of $1 \mathrm{C}$ for 200 cycles, and the specific capacities of the thin-film electrodes were calculated based on the total masses of the $\mathrm{Ge}$ or $\mathrm{Ge}-\mathrm{Al}$ electrodes. Fig. 5 shows the reversible capacity of pure $\mathrm{Ge}$ and $\mathrm{Ge}-\mathrm{Al}$ multilayers as a function of cycle number. As observed in Fig. 5, all Ge-Al multilayer samples exhibit higher specific capacity and better cyclability than the pure Ge electrode. Among the Ge-Al multilayers, Ge-Al-20 shows the highest capacity of 1352 $\mathrm{mAh} \mathrm{g}^{-1}$; this high capacity is maintained stably over 200 cycles. Accordingly, the capacity of Ge-Al-20 after 200 cycles is recorded as $\sim 1382 \mathrm{mAh} \mathrm{g}^{-1}$, corresponding to almost $100 \%$ capacity retention. The superior electrochemical performance of the $\mathrm{Ge}-\mathrm{Al}$ multilayers is attributed to the Al layers, which act as elastic layers that absorb the internal stresses induced during cycling and facilitate the $\mathrm{Li}$ ion diffusion in the bulk Ge-Al multilayer electrode. The flexibility of $\mathrm{Al}$ and the high Li diffusivity of the Ge sand-
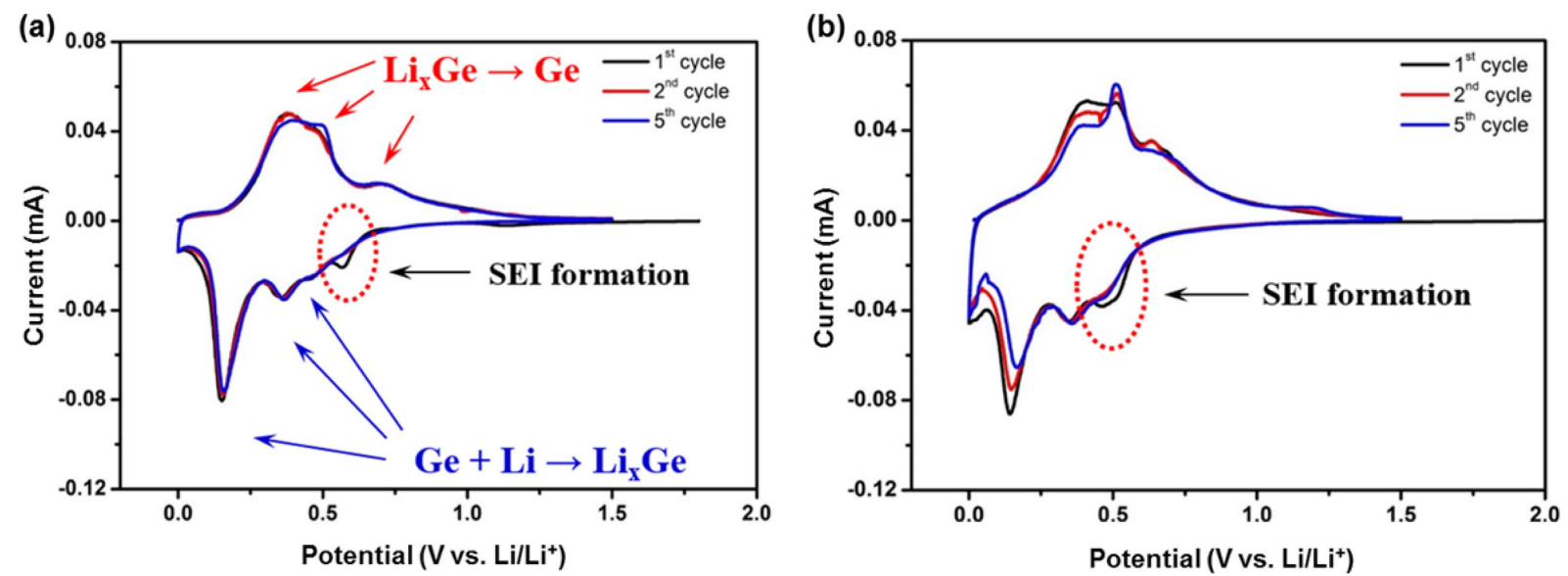

Fig. 4. Cyclic voltammograms (CVs) of the (a) Ge and (b) Ge-Al-20 electrodes, respectively, measured under the scan rate of 0.05 $\mathrm{mV} \mathrm{s}^{-1}$. 

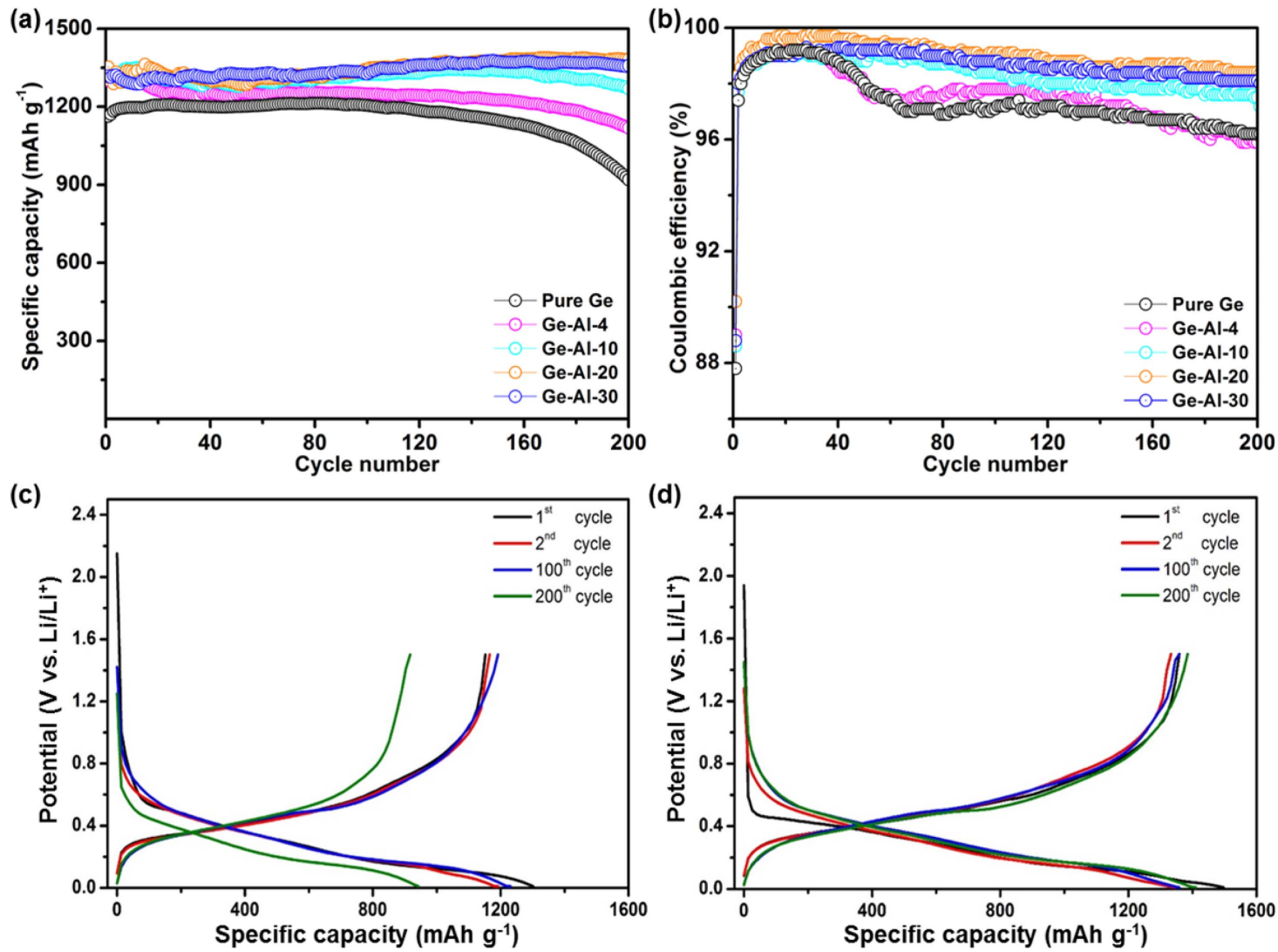

Fig. 5. (a) Cyclability and (b) coulombic efficiency of the Ge-Al multilayer and pure Ge electrodes measured under a rate of $1 \mathrm{C}$; selected potential profiles of (c) Ge and (d) Ge-Al-20.

wiched by $\mathrm{Al}$ permit the maintenance of intimate contact with Ge while regulating the $\mathrm{Li}$ supplied for electrochemical reaction. Therefore, better electrochemical performance is achieved for the Ge-Al multilayers. On the contrary, the pure Ge film electrode shows poorer electrochemical performance. For instance, in the first cycle the pure Ge has a capacity of $1163 \mathrm{mAh} \mathrm{g}^{-1}$, which gradually increases through an activation process after some cycling. The specific capacity of pure Ge stabilizes after five cycles and is maintained at a high value for $\sim 90$ cycles. Then the capacity of pure Ge gradually declines. After 200 cycles, the capacity of the pure Ge electrode is only $920 \mathrm{mAh} \mathrm{g}^{-1}$, corresponding to $79 \%$ capacity retention. The rapid capacity fading of pure Ge occurs because of the unstable SEI layer on the surface of Ge.

We determined that Ge-Al-20 was the optimal sample among the $\mathrm{Ge}-\mathrm{Al}$ multilayer electrodes; hence, it is used for further analysis along with pure Ge electrodes. The formation of SEI layers is attributed to the decomposition of the electrolyte on the electrode surface at low potentials. Because of the huge volume expansion accompanying repeated cycling, the Ge electrode is pulverized and a new SEI forms on the nascent Ge surface. This process is repeated until Ge is pulverized to its minimum particle size, causing capacity fading. Fortunately, for the $\mathrm{Ge}-\mathrm{Al}$ multilayers, the covering of $\mathrm{Ge}$ with an $\mathrm{Al}$ layer inhibits the formation of the SEI layer. Consequently, the cyclability of $\mathrm{Ge}-\mathrm{Al}$ is superior over that of pure Ge. The formation of the SEI layer also affects the coulombic efficiency of the pure Ge electrode, as seen in Fig. 5(b). In the first cycle, the coulombic efficiency of pure Ge is $\sim 87.8 \%$, the lowest among those of the investigated samples. In the next cycles, the coulombic efficiency is rapidly improved, reaching the highest value of $99.2 \%$ in the $26^{\text {th }}$ cycle. However, afterward, the coulombic efficiency of the pure Ge rapidly decays. This can be attributed to the failure of the SEI layer, causing the consumption of more $\mathrm{Li}$ in side reactions. Similar behavior is observed for the GeAl-4 sample. The thicker layer of Ge induces dramatic volume expansion during lithiation, causing the breakdown of the $\mathrm{Al}$ protection layer. The attempt to minimize the thickness of $\mathrm{Ge}$ and $\mathrm{Al}$ in order to improve the performance of the $\mathrm{Ge}-\mathrm{Al}$ multilayer fails. For Ge-Al-30, the coulombic efficiency in the first cycles is $\sim 88.8 \%$. This value is smaller compared to the $90.2 \%$ efficiency of $\mathrm{Ge}-\mathrm{Al}-20$. The average coulombic efficiencies of $\mathrm{Ge}-\mathrm{Al}-20$ and $\mathrm{Ge}-\mathrm{Al}-30$ over 200 cycles are 99.08 and $98.81 \%$, respectively. As the $\mathrm{Al}$ film becomes thinner, defects can appear. Therefore, $\mathrm{Al}$ cannot protect Ge well, permitting the electrolyte to attach easily to Ge and form an unstable SEI layer. This is why Ge-Al-30 shows poorer electrochemical performance compared to Ge- 


\section{Al-20.}

Figure 5(c), d shows the potential profiles of pure Ge and Ge-Al-20 multilayers. For the pure Ge cell, when the current is applied, the electrode potential suddenly drops to 0.8 $\mathrm{V}$ vs. $\mathrm{Li} / \mathrm{Li}^{+}$. In the next step, two main plateaus are located at 0.37 and $0.12 \mathrm{~V}$ vs. $\mathrm{Li} / \mathrm{Li}^{+}$, ascribed to the lithiation process of the Ge film. In the first cycle, we recorded the discharge capacity of $1324 \mathrm{mAh} \mathrm{g}^{-1}$ and the charge capacity of $1163 \mathrm{mAh} \mathrm{g}^{-1}$ for the pure Ge film. This means that $\sim 161$ $\mathrm{mAh} \mathrm{g}^{-1}$ is consumed in the irreversible capacity of the first cycle. Unlike highly crystalline Ge, the obtained amorphous Ge shows higher potential for lithiation in the first cycle. This phenomenon originates from the amorphous nature of the material and the open structure enabling easy lithiation. Notably, the discharge and charge curves from the first and $100^{\text {th }}$ cycles are perfectly overlapped. However, because of capacity fading from the $100^{\text {th }}$ cycle onward, the discharge and charge curves of the $200^{\text {th }}$ cycle are quite different from the others. Meanwhile, for Ge-Al-20, the discharge and charge curves for the first and $200^{\text {th }}$ cycles are quite similar. This suggests the superior stability of $\mathrm{Ge}-\mathrm{Al}-20$. In the first cycle, the discharge and charge capacities of Ge-Al-20 are 1499 and $1353 \mathrm{mAh} \mathrm{g}^{-1}$, respectively. After 200 complete cycles, the $\mathrm{Ge}-\mathrm{Al}-20$ electrode maintains a capacity of 1381 $\mathrm{mAh} \mathrm{g}^{-1}$.

To investigate the morphology change upon cycling, the coin cells containing Ge and Ge-Al-20 electrodes after 100 and 200 cycles were dis-assembled, the Ge and Ge-Al-20 electrodes were collected, and the electrodes were examined by SEM. Fig. 6 shows the morphologies of Ge and Ge-Al-20 after 100 and 200 cycles. The microstructural changes of pure Ge are shown in the SEM images (Fig. 6(a), (b)). Many cracks are observed on the surface of pure Ge after 100 cycles, resulting from the volume expansion associated with the continuous growth of the SEI layer during cycling. The electrode is severely damaged after 200 cycles, with cracks becoming larger, exposure of the copper substrate, and peeloff of the active material from the current collector. This causes the dramatic capacity fading. Fig. 6(d) shows the surface morphology of Ge-Al-20 after 100 and 200 cycles, respectively. For the Ge-Al-20 electrode, a compact and flat surface is maintained after 100 cycles, compared to the pure Ge electrode. The SEM image of Ge-Al-20 after 200 cycles (Fig. 6(e)) shows some cracks on the surface. Compared to pure Ge (Fig. 6(b)), these cracks can be attributed to the expansion of Ge during cycling, which is how, even after 200 cycles, the specific capacity of $\mathrm{Ge}-\mathrm{Al}-20$ is almost unchanged. By adding the $\mathrm{Al}$ buffer layers, even after changes in the morphology of $\mathrm{Ge}-\mathrm{Al}$, the electrical contact of the active material is maintained, thereby preserving the electrochemical performance. In the multilayer electrode, the fading capacity of Ge caused by pulverization, agglomeration, and loss of contact during the charging and discharging processes is completely addressed. In addition, the surface of
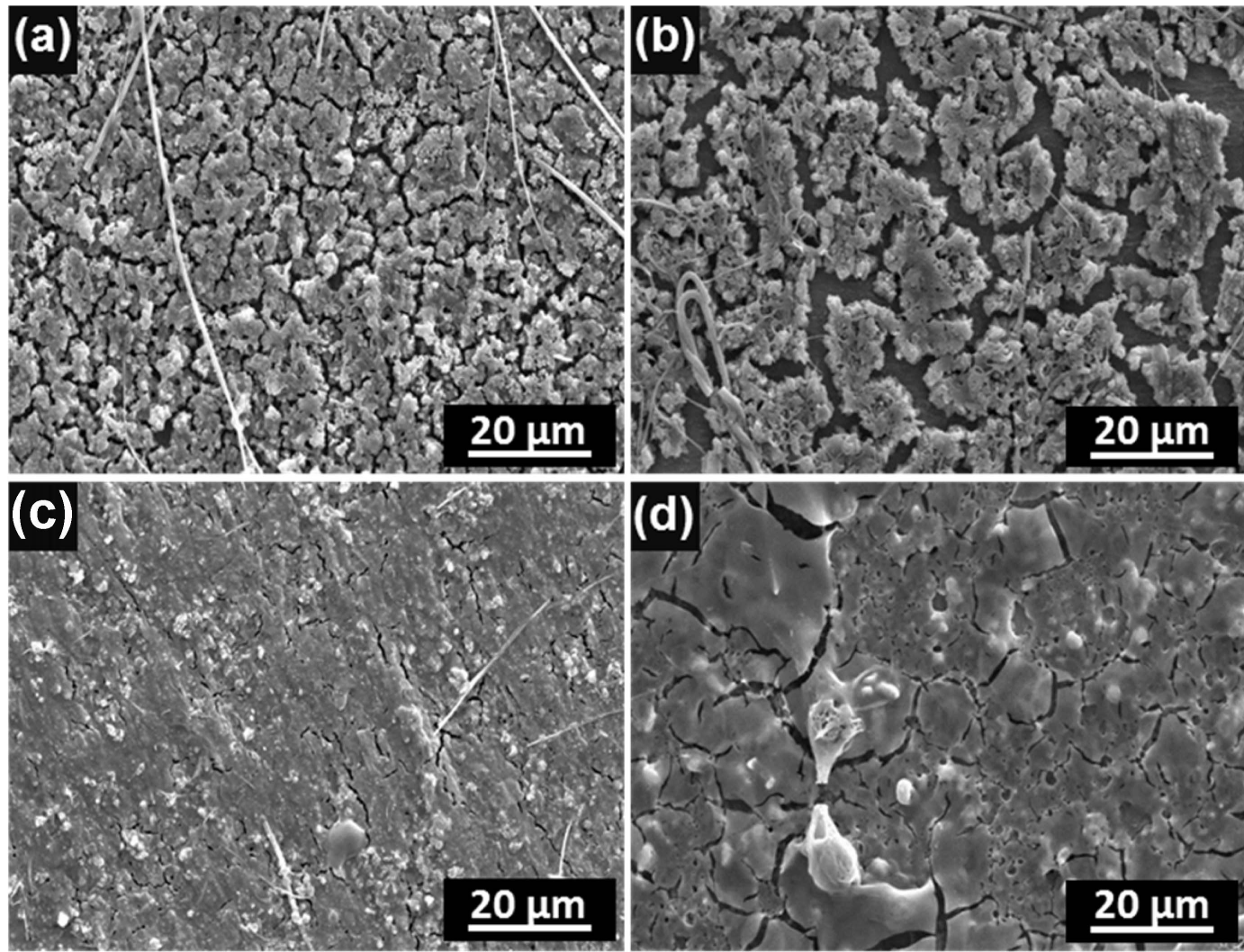

Fig. 6. Surface morphologies of the Ge electrode after (a) 100 and (b) 200 cycles, respectively; surface morphologies of the Ge-Al20 electrode after (c) 100 and (b) 200 cycles, respectively. 


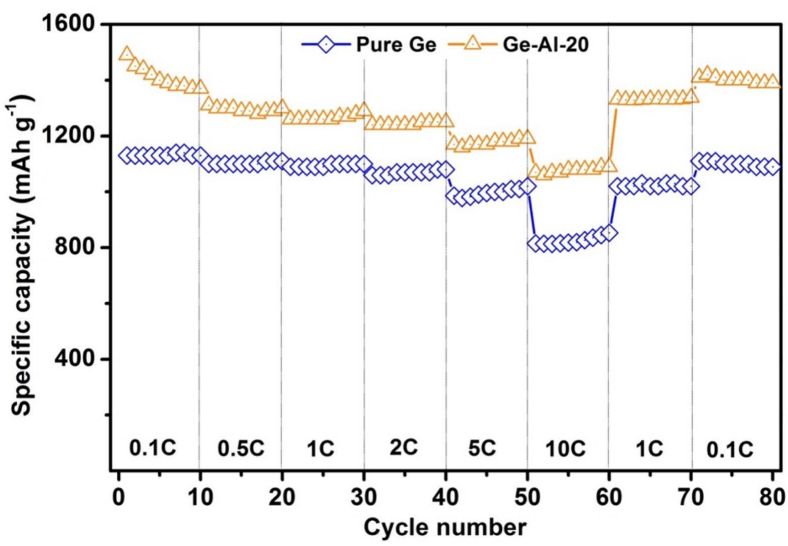

Fig. 7. Rate capabilities of the Ge and Ge-Al-20 electrodes.

Ge-Al-20 seems to be covered with an SEI layer generated during the charge and discharge processes. The optical images of $\mathrm{Ge}-\mathrm{Al}-20$ after 100 and 200 cycles show the original shape with no damage.

To verify the electrochemical performances of the pure Ge and Ge-Al-20 multilayers, the rate capabilities of both electrodes were measured at $0.1 \mathrm{C}, 0.5 \mathrm{C}, 1 \mathrm{C}, 2 \mathrm{C}, 5 \mathrm{C}$, and $10 \mathrm{C}$, before returning to $0.1 \mathrm{C}$ for ten cycles at each rate step. As seen in Fig. 7, the cycling performance of the Ge-Al-20 multilayer thin film anode is relatively stable at each discharge rate; the electrode maintains a specific capacity of 1179 $\mathrm{mAh} \mathrm{g}^{-1}$ at the $10 \mathrm{C}$ rate. The specific capacity of $\mathrm{Ge}-\mathrm{Al}-20$ is decreased by only $\sim 17 \%$ when the charge and discharge rate increases by 10 times. Upon returning to the rate of $0.1 \mathrm{C}$, the specific capacity recovers the original value of $1367 \mathrm{mAh} \mathrm{g}^{-1}$, demonstrating that this material can be operated at higher C-rates. On the other hand, the capacity of the pure Ge electrode is decreased continuously with increases in the C-rate. The decrease in capacity at higher rates can be attributed to the relatively poor electronic conductivity of the pure Ge and the limitation of Li diffusivity in pure Ge. The reversible capacities of the Ge-Al-20 electrode are 1432, 1367, 1315, 1273, 1267, 1242, and $1179 \mathrm{mAh}$ $\mathrm{g}^{-1}$ at rates of $0.1,0.5,1,2,5$, and $10 \mathrm{C}$, respectively. This suggests that the Ge-Al-20 electrode possesses excellent rate capability. On the contrary, for the pure Ge electrode at $0.1 \mathrm{C}$, the specific capacity of $1229 \mathrm{mAh} \mathrm{g}^{-1}$ is measured. However, when the charge and discharge rate increases, the specific capacity drops considerably compared to that of the Ge-Al-20 electrode. At the rate of $10 \mathrm{C}$, the specific capacity of pure Ge is only $~ 886 \mathrm{mAh} \mathrm{g}^{-1}$. The superiority of $\mathrm{Ge}-\mathrm{Al}$ 20 over the pure Ge electrode in rate capability measurements can be attributed to the presence of $\mathrm{Al}$ in the sample. $\mathrm{Al}$ possesses high electrical conductivity and high Li diffusivity in the matrix, allowing Li-ions to penetrate the electrode and thereby enhancing the Li diffusion. In addition, the thin-film electrode has an amorphous structure that promotes Li-ion transfer, allowing better rate capability. Finally, the presence of $\mathrm{Al}$ between Ge layers reduces the resistance of the Ge-Al-20 electrode, decreasing the Ohmic polarization. Therefore, the $\mathrm{Ge}-\mathrm{Al}-20$ multilayer shows better rate capability than pure Ge.

\section{Conclusions}

A Ge-Al multilayer film anode was successfully fabricated by an RF sputtering system. Among the Ge-Al multilayer samples, the Ge-Al-20 electrode showed the best electrochemical performance compared to the other multilayer samples and the pure Ge film. At the rate of $1 \mathrm{C}$, the $\mathrm{Ge}-\mathrm{Al}-$ 20 electrode showed better capacity retention; after 200 cycles, the specific capacity of Ge-Al-20 was $1382 \mathrm{mAh} \mathrm{g}^{-1}$, corresponding to approximately $100 \%$ of the initial capacity. At the high rate of $10 \mathrm{C}$, the Ge-Al-20 showed a capacity of $1197 \mathrm{mAh} \mathrm{g}^{-1}$. The superiority of the Ge-Al-20 electrode over the pure Ge electrode is attributed to the $\mathrm{Al}$ buffer layer in the multilayer structure. The $\mathrm{Al}$ layers act as elastic buffer layers to mitigate the stress inside the material and improve the conductivity of the active material, thereby achieving better cyclability and rate capability. In addition, because of the stability of the $\mathrm{Al}$ layer in contact with the electrolyte, SEI formation during cycling was inhibited, which preserved the integrity of the Ge film. Finally, the improved cyclability, rate capability, and capacity of the Ge-Al-20 electrode demonstrated that the multilayer approach is effective in improving the electrochemical performance of Ge films.

\section{Acknowledgments}

This research was supported by the National Research Foundation (NRF) of Korea grant (No. 2015R1D1A3A01019399 and No. 2016R1A2B4015883).

\section{REFERENCES}

1. M. Armand and J.-M. Tarascon, "Building Better Batteries," Nature, 451 [7179] 652-57 (2008).

2. Y.-H. Lee, J.-S. Kim, J. Noh, I. Lee, H. J. Kim, S. Choi, J. Seo, S. Jeon, T.-S. Kim, J.-Y. Lee, and J. W. Choi, "Wearable Textile Battery Rechargeable by Solar Energy," Nano Lett., 13 [11] 5753-61 (2013).

3. S.-H. Kim, K.-H. Choi, S.-J. Cho, S. Choi, S. Park, and S.-Y. Lee, "Printable Solid-State Lithium-Ion Batteries: A New Route toward Shape-Conformable Power Sources with Aesthetic Versatility for Flexible Electronics," Nano Lett., 15 [8] 5168-77 (2015).

4. E. Peled, C. Menachem, D. Bar-Tow, and A. Melman, "Improved Graphite Anode for Lithium-Ion Batteries Chemically: Bonded Solid Electrolyte Interface and Nanochannel Formation,” J. Electrochem. Soc., 143 [1] L4-7 (1996).

5. Q. Pan, K. Guo, L. Wang, and S. Fang, "Novel Modified Graphite as Anode Material for Lithium-Ion Batteries," J. Electrochem. Soc., 149 [9] A1218-23 (2002).

6. H. Song, H. X. Wang, Z. Lin, X. Jiang, L. Yu, J. Xu, Z. Yu, X. Zhang, Y. Liu, P. He, L. Pan, Y. Shi, H. Zhou, and K. Chen, "Highly Connected Silicon-Copper Alloy Mixture 
Nanotubes as High-Rate and Durable Anode Materials for Lithium-Ion Batteries," Adv. Funct. Mater., 26 [4] 524-31 (2016).

7. Y.-C. Zhang, Y. You, S. Xin, Y.-X. Yin, J. Zhang, P. Wang, X. Zheng, F.-F. Cao, and Y.-G. Guo, "Rice Husk-Derived Hierarchical Silicon/Nitrogen-Doped Carbon/Carbon Nanotube Spheres as Low-Cost and High-Capacity Anodes for Lithium-Ion Batteries," Nano Energy, 25 120-27 (2016).

8. D. T. Ngo, H. T. T. Le, C. Kim, J.-Y. Lee, J. G. Fisher, I.-D. Kim, and C.-J. Park, "Mass-Scalable Synthesis of 3D Porous Germanium-Carbon Composite Particles as an Ultra-High Rate Anode for Lithium Ion Batteries," Energy Environ. Sci., 8 [12] 3577-88 (2015).

9. J. Y. Huang, L. Zhong, C. M. Wang, J. P. Sullivan, W. Xu, L. Q. Zhang, S. X. Mao, N. S. Hudak, X. H. Liu, A. Subramanian, H. Fan, L. Qi, A. Kushima, and J. Li, "In Situ Observation of the Electrochemical Lithiation of a Single $\mathrm{SnO}_{2}$ Nanowire Electrode," Science, 330 [6010] 1515-20 (2010).

10. K. C. Klavetter, S. M. Wood, Y.-M. Lin, J. L. Snider, N. C. Davy, A. M. Chockla, D. K. Romanovicz, B. A. Korgel, J.-W. Lee, A. Heller, and C. B. Mullins, "A High-Rate Germanium-Particle Slurry Cast Li-Ion Anode with High Coulombic Efficiency and Long Cycle Life," J. Power Sources, 238 123-36 (2013).

11. D. T. Ngo, R. S. Kalubarme, H. T. T. Le, J. G. Fisher, C.-N. Park, I.-D. Kim, and C.-J. Park, "Carbon-Interconnected Ge Nanocrystals as an Anode with Ultra-Long-Term
Cyclability for Lithium Ion Batteries," Adv. Funct. Mater., 24 [33] 5291-98 (2014).

12. N. Nitta and G. Yushin, "High-Capacity Anode Materials for Lithium-Ion Batteries: Choice of Elements and Structures for Active Particles," Part. Part. Syst. Charact., 31 [3] 317-36 (2014).

13. D. T. Ngo, H. T. T. Le, R. S. Kalubarme, J.-Y. Lee, C.-N. Park, and C.-J. Park, "Uniform $\mathrm{GeO}_{2}$ Dispersed in Nitrogen-Doped Porous Carbon Core-Shell Architecture: An Anode Material for Lithium Ion Batteries," J. Mater. Chem. A, 3 [43] 21722-32 (2015).

14. D. T. Ngo, R. S. Kalubarme, H. T. T. Le, C.-N. Park, and C.J. Park, "Conducting Additive-Free Amorphous $\mathrm{GeO}_{2} / \mathrm{C}$ Composite as a High Capacity and Long-Term Stability Anode for Lithium Ion Batteries," Nanoscale, 7 [6] 2552-60 (2015).

15. C. Gong, D. Ruzmetov, A. Pearse, D. Ma, J. N. Munday, G. Rubloff, A. A. Talin, and M. S. Leite, "Surface/Interface Effects on High-Performance Thin-Film All-Solid-State LiIon Batteries," ACS Appl. Mater. Interfaces, 7 [47] 2600711 (2015).

16. B. Laforge, L. Levan-Jodin, R. Salot, and A. Billard, "Study of Germanium as Electrode in Thin-Film Battery," J. Electrochem. Soc., 155 [2] A181-88 (2008).

17. L. Baggetto and P. H. L. Notten, "Lithium-Ion (De)Insertion Reaction of Germanium Thin-Film Electrodes: An Electrochemical and In Situ XRD Study," J. Electrochem. Soc., 156 [3] A169-75 (2009). 